LAWRENCE LIVERMORE N A TION AL LABORATORY
Anisotropy in magnetic and
transport properties of GdCrSb3

D. D. Jackson, Z. Fisk

December 17, 2003

Chemistry of Materials 
This document was prepared as an account of work sponsored by an agency of the United States Government. Neither the United States Government nor the University of California nor any of their employees, makes any warranty, express or implied, or assumes any legal liability or responsibility for the accuracy, completeness, or usefulness of any information, apparatus, product, or process disclosed, or represents that its use would not infringe privately owned rights. Reference herein to any specific commercial product, process, or service by trade name, trademark, manufacturer, or otherwise, does not necessarily constitute or imply its endorsement, recommendation, or favoring by the United States Government or the University of California. The views and opinions of authors expressed herein do not necessarily state or reflect those of the United States Government or the University of California, and shall not be used for advertising or product endorsement purposes. 


\title{
UCRL-JRNL-201517
}

\section{Anisotropy in magnetic and transport properties of $\mathrm{GdCrSb}_{3}$}

\author{
D. D. Jackson \\ Lawrence Livermore National Laboratory, Livermore, CA 94550 \\ Z. Fisk
}

National High Magnetic Field Laboratory, Tallahassee, FL 32310

\begin{abstract}
We report the first measurements of anisotropy in magnetic susceptibility, magnetization, and electrical resistivity using single crystals of $\mathrm{GdTSb}_{3}(T=\mathrm{Cr}, \mathrm{V})$. $\mathrm{GdTSb}_{3}$ is a quasi-two dimensional system with orthorhombic crystal structure (space group $P b c m)$. Unlike the other light rare earth chromium antimonides $(R=$ Ce-Nd, Sm), in which two magnetic transitions are observed, $\mathrm{GdCrSb}_{3}$ undergoes a single ferrimagnetic transition at $T_{C}=86 \mathrm{~K}$, which is evident in both the magnetic susceptibility and electrical resistivity. Within the $b-c$ plane, $\mathrm{GdCrSb}_{3}$ is found to have metallic behavior from $5 \mathrm{~K}$ to $300 \mathrm{~K}$, but it is found to have insulating behavior $\left(d \rho_{a} / d T<0\right)$ along the stacking axis. $\mathrm{GdVSb}_{3}$ is found to have a Néel transition at $5 \mathrm{~K}$ due to the localized Gd ions, but no ferromagnetic transition.
\end{abstract}

Key words: magnetism, antimonides, resistivity, $R \mathrm{CrSb}_{3}$

PACS: $75.30 . \mathrm{Gw}, 72.80 . \mathrm{Ga}, 72.15 .-\mathrm{v}, 75.30 . \mathrm{Cr}$

Preprint submitted to Elsevier Science

11 February 2004 


\section{Introduction}

The family of compounds, $R \mathrm{CrSb}_{3}(R=\mathrm{La}-\mathrm{Nd}$, Sm, Gd-Dy), have had many investigations in the literature due to their unique magnetic and electrical transport properties[1-12]. The parent member in the series, $\mathrm{LaCrSb}_{3}$, has been studied in depth [3-5] because it has only one magnetic ion, Cr, yet it has a very complex magnetic phase diagram. It has been found to have a Curie temperature at $T_{C}=132 \mathrm{~K}$, followed by a spin reorientation phase for $T<98 \mathrm{~K}$ and $H<250 \mathrm{G}$ due to the unconventional magnetism involving the coexistence of local and itinerant $\mathrm{Cr}$ moments.[4,5] While the itinerant ferromagnetic (FM) phase continues to exist for $R \mathrm{CrSb}_{3}(R=\mathrm{Ce}-\mathrm{Nd}, \mathrm{Sm})$, a second low temperature magnetic phase occurs due to the ordering of the rare-earth ions.[6-10] $\mathrm{SmCrSb}_{3}$ was found to be unique in the series due to the presence of a first order AFM transition at $30 \mathrm{~K}$ due to the ordering of the Sm ions.[10] Polycrystalline samples of $R=\mathrm{Gd}$, Tb, and Dy have been investigated, $[7,12]$ which show only one AFM transition, but the value of the transition temperature is still debated. Single crystal electrical resistivity measurements have also been collected along the $c$-axis, [12] but to our knowledge, this is the first report of the magnetization and electrical resistivity along all three principle axis of $\mathrm{GdCrSb}_{3}$ using high quality single crystals.

\section{Experimental Technique}

Single crystals of $\mathrm{GdTSb}_{3}$ were prepared from ingots of the elements [Gd from Ames Laboratory; Cr (99.996\%), V (99.5\%), and Sb (99.99\%) from Alfa

Æsar], and the growth procedure has been previously described[4]. GdTSb 3 
crystallizes in an orthorhombic structure (space group Pbcm), $[1,2]$ and consists of two distinct planar layers of $\mathrm{Cr}$ and $\mathrm{Sb}$ perpendicular to the $a$-axis which are separated by Gd ions (see Fig. 1a). The first of these layers consists of chains of $\mathrm{Cr}$ atoms extending along the $c$ direction, with $\mathrm{Sb}$ atoms forming face sharing (edge sharing) octahedra along the $c$-axis (b-axis). The Gd ions lie in a checker board type pattern which alternates above and below the Sb plane. This quasi-2D crystal structure suggests that anisotropy may play a crucial role in understanding its properties. The lattice parameters were determined using a commercial Scintag x-ray diffractometer using a Si standard, and a least squares fit to a minimum of 20 peaks. The lattice constants for $\mathrm{GdCrSb}_{3}$ were determined to be $a=12.75(6) \AA, b=6.15(2) \AA$, and $c=6.01(4) \AA$, and

for $\mathrm{GdVSb}_{3}, a=12.82(2) \AA, b=6.22(3) \AA$, and $c=5.96(2) \AA$.

Magnetization and magnetic susceptibility measurements were taken with a commercial superconducting quantum interference device (SQUID) magnetometer (Quantum Design MPMSR2) in the temperature range 2-350 $\mathrm{K}$ and $-55 \mathrm{kG} \leq H \leq 55 \mathrm{kG}$. High field magnetization measurements were collected using a vibrating sample magnetometer in a field range of $0 \leq H \leq 31 \mathrm{~T}$ at the National High Magnetic Field Laboratory. Electrical resistivity measurements were performed using a standard four probe technique in the temperature range 5-295 K. All measurements were collected on as grown samples.

\section{Magnetization}

One expects $\mathrm{GdCrSb}_{3}$ to have somewhat different properties than the light rare-earth antimonides in the series because $\mathrm{Gd}^{3+}$ has a half full $f$-electron shell, and therefore has zero orbital angular momentum and a large effective 
moment $\left(\mu_{e f f}=7.94 \mu_{B}\right.$ according to Hund's Rules). Fig. 1 shows the fieldcooled magnetization as a function of temperature with an applied field of $1 \mathrm{kG}$. Unlike the previous members in the series, there is no evidence of a FM phase for $\mathrm{GdCrSb}_{3}$, but it does undergo a magnetic transition at $T_{C}=86 \mathrm{~K}$. This is most easily observed as a peak along the $c$-axis, and is consistent with this being the easy axis of a ferrimagnetic phase, and is the only apparent magnetic phase transition. Fig. 1 also shows an upturn in the susceptibility at low temperatures along both the $a$ and $b$-axis, which may be due to the Cr vacancies which are known to exist in these compounds. [5] These vacancies may play a role in the discrepancies in the reported transition temperatures. For example, Deakin et al. report a value of $92 \mathrm{~K}$ for the magnetic transition,[12] and Leonard et al. report a value of $26 \mathrm{~K} .[7]$

The high temperature inverse susceptibility $(T>200 \mathrm{~K})$ was fit to a modified Curie-Weiss law, $1 /\left(\chi-\chi_{0}\right)=(T-\theta) / C$, where $\chi_{0}$ is the temperature independent susceptibility, $\theta$ is the Curie temperature, and $C$ is the Curie constant. The data is shown in Fig. 1b, with the values from the fit given in Table 1 . The expected value of $\chi_{0}$ can be approximated to be approximately $-1 \times 10^{-4} \mathrm{emu} / \mathrm{mol}[13]$, which is an order of magnitude larger than what is found for this system. This, as well as the metallic electrical resistivity, is consistent with itinerant behavior for the $R \mathrm{CrSb}_{3}$ family. Table 1 also lists the effective moment of $\mathrm{GdCrSb}_{3}$ for each axis. A polycrystalline average gives $\mu_{e f f}=9.21 \mu_{B} /$ f.u., which is larger than the value predicted by Hund's Rules, but close to the value of $8.16 \mu_{B} /$ f.u., obtained by Deakin et al..[12] They suggest that this large value is an indication of the strong interactions of the $3 d$ and $4 f$ moments above the ordering temperature. The downward curvature seen in the inverse susceptibility near $T_{C}$ (Fig. 1b) is the expected behavior 
for a ferrimagnetic system.

The magnetization as a function of applied magnetic field (Fig. 2) also suggests that below $T_{C}=86 \mathrm{~K}, \mathrm{GdCrSb}_{3}$ is ferrimagnetic. At $5 \mathrm{~K}$, one finds that $M_{a}$ and $M_{b}$ linearly increase with increasing field, while $M_{c}$ undergoes a spin-flop transition at $26 \mathrm{kG}$, which implies that the easy axis is parallel to the $\mathrm{Cr}$ chains (the $c$-axis). The inset to Fig. 2 shows the magnetization along the $a$ axis up to $31 \mathrm{~T}$ at $10 \mathrm{~K}$ and $75 \mathrm{~K}$. The $a$-axis magnetization begins to saturate just below $15 \mathrm{~T}$ to a value of $5.6 \mu_{B} /$ f.u., which is smaller than the predicted saturation value of $\mathrm{Gd}^{3+}\left(\sigma=g J=7 \mu_{B}\right)$.

\section{Electrical Resistivity}

The distinctive behavior of the magnetization leads one to expect unique electrical resistivity as well, and Fig. 3 shows that this is found to be true. The initial high temperature behavior of the electrical resistivity is similar to both $\mathrm{LaCrSb}_{3}$ and $\mathrm{SmCrSb}_{3},[4,10]$ with positive temperature dependence for the in-plane $(b-c$ plane) resistivity, and non-metallic $(d \rho / d T<0)$ temperature dependence for the out of plane ( $a$-axis) resistivity. Below $T_{C}$, the in-plane resistivity continues to have a positive slope. While the residual resistivity ratio for both axis $\left(R R R_{b}=0.45, R R R_{c}=0.70\right)$ is small, the values are larger than what has been observed in other members of the series. For example it is ap-

proximately three times larger than $\mathrm{LaCrSb}_{3}\left(R R R_{b}=0.16, R R R_{c}=0.23\right),[4]$ and twice as large as $\mathrm{SmCrSb}_{3}\left(R R R_{b}=0.19, R R R_{c}=0.34\right) .[10]$ This small value is likely due to the large number of Cr vacancies, such as has been observed for $\mathrm{LaCrSb}_{3} \cdot[5]$ For $R=\mathrm{La}-\mathrm{Nd}$, and $\mathrm{Sm}$, the resistivity along the $a$-axis (the stacking axis) was found to have a change in the sign of the slope below 
$T_{C}$, but for $\mathrm{GdCrSb}_{3}$, a kink is observed, below which the resistivity continues to rise with an increased slope. Fig. 3a shows that a Fisher-Langer-type anomaly is present along the $b$-axis (as well as the $c$-axis, data not shown), only the peak has become broadened compared with $\mathrm{LaCrSb}_{3}$ and $\mathrm{SmCrSb}_{3}$. Below $T_{C}$, Fig. 3b shows that the in-plane resistivity decreases as $T^{3 / 2}$, which is the same behavior found for both $R=\mathrm{La}[4]$ and Sm.[10] This temperature dependence for the electrical resistivity is common for a FM systems in which the scattering length is reduced from an ideal system,[14] which suggests that the in-plane scattering mechanism for $\mathrm{GdCrSb}_{3}$ is similar to that found for $R=$ La and $\mathrm{Sm}$.

\section{$5 \mathrm{GdVSb}_{3}$}

$\mathrm{LaVSb}_{3}$ was found to be a non-magnetic counterpart to $\mathrm{LaCrSb}_{3}$. Therefore, in order to understand the properties of $\mathrm{GdCrSb}_{3}$ in the absence of itinerant magnetism, isostructural $\mathrm{GdVSb}_{3}$ was investigated. The in-plane anisotropy in the magnetization for $\mathrm{GdVSb}_{3}$ was found to be zero. Therefore Fig. 4a shows only the data along the $c$-axis, with a modified Curie-Weiss fit between $45 \mathrm{~K}$ and $330 \mathrm{~K}$, and the resulting parameters given in Table 1 . The low temperature susceptibility is shown in the inset to Fig. 4a in which a peak is

found at $T_{N}=5 \mathrm{~K}$. This is very similar behavior to that seen in $\mathrm{SmVSb}_{3} \cdot[10]$ The values obtained are close to the expected value for $\mathrm{Gd}^{3+}$ of $\mu_{\text {eff }}=7.94 \mu_{B}$.

The electrical resistivity normalized at room temperature is shown in Fig. 4b. Both axes have similar behavior to what was found for $\mathrm{SmVSb}_{3},[10]$ only the $\mathrm{AFM}$ ordering temperature for $\mathrm{GdVSb}_{3}$ is large enough that an initial increase in the resistivity just above $5 \mathrm{~K}$ can be seen. Due to the small thickness, it 
was not possible to measure the electrical resistivity along the $a$-axis.

\section{Discussion}

$\mathrm{GdVSb}_{3}$ is isostructural to $\mathrm{GdCrSb}_{3}$, but, like $\mathrm{LaVSb}_{3}$, in which the magnetic susceptibility is small and temperature independent due to the absence of itinerant magnetism, [4] the magnetic susceptibility of $\mathrm{GdVSb}_{3}$ is due only to the localized $4 f$-electrons of $\mathrm{Gd}^{3+}$. It is magnetically isotropic, unlike $\mathrm{GdCrSb}_{3}$, and it has a Néel temperature near $5 \mathrm{~K}$ due to ordering of the $4 f$-moments. This points to the importance of the interactions between the itinerant $3 d$ electrons and the localized $4 f$-electrons in determining the properties of the $\mathrm{RCrSb}_{3}$ family of materials.

$R \mathrm{CrSb}_{3}(R=\mathrm{Ce}-\mathrm{Nd}$, and $\mathrm{Sm})$ are all found to undergo two magnetic transitions due to the itinerant FM phase of the $3 d$-electrons at high temperatures, followed by a low temperature ordering of the localized rare-earth ions. [6-10] For $R=\mathrm{Sm}$ and $\mathrm{Gd}$, the interactions between the itinerant $3 d$ and localized $4 f$-electrons are strong enough to bring about unique properties in these two materials. $\mathrm{SmCrSb}_{3}$ was found to undergo a first order Néel transition at $30 \mathrm{~K}$, and isothermal magnetization hysteresis loops observed at low fields below $T_{N}$ suggest that the itinerant $3 d$-electrons continue to exhibit FM interactions, but the localized $4 f$-moments nearly cancel out the macroscopic magnetiza-

tion of the sample.[10] Although our magnetization results can not probe the microscopic behavior of the $3 d$ and $4 f$-moments, it would be consistent with the $\mathrm{SmCrSb}_{3}$ results for the compound to be described by two independent, yet heavily interacting subsystems: one composed of itinerant $3 d$-electrons, and a second of anti-aligned localized $4 f$-moments. 
It is believed that similar behavior is found for $\mathrm{GdCrSb}_{3}$, but for this material, both magnetic lattices order at the same temperature and appear to be antiparallel, resulting in a complex ferrimagnetic ordering. The downward curvature in $1 / \chi$ just above $T_{C}$ (Fig. 1b) is indicative of ferrimagnetism. Recent ac magnetic susceptibility measurements of $\mathrm{GdCrSb}_{3}[12]$ show a significant imaginary component to the magnetic susceptibility, which provides further support for a ferrimagnetic phase. The spin-flop transition at $26 \mathrm{kG}$ and $5 \mathrm{~K}$ is also consistent with a ferrimagnetic phase, and indicates the easy axis is along the $c$-axis. At $30 \mathrm{~K}$, Deakin et al.[12] observed a maximum in the magnetoresistance at $30 \mathrm{kG}$ when the magnetic field was oriented along the c-axis, and no maximum with the magnetic field aligned perpendicular to the c-axis. The inplane $T^{3 / 2}$ resistivity behavior of $\mathrm{GdCrSb}_{3}$ is also similar to both $\mathrm{LaCrSb}_{3}$ and $\mathrm{SmCrSb}_{3}$, suggesting that the scattering mechanisms are similar, which would again be consistent with itinerant ferromagnetism due to the $3 d$-electrons below $T_{C}=86 \mathrm{~K}$, but strong interactions with the $4 f$-moments resulting in an overall ferrimagnetic phase.

The transition temperatures for $R \mathrm{CrSb}_{3}(R=\mathrm{La}-\mathrm{Nd}, \mathrm{Sm}$, and $\mathrm{Gd})$ are plotted vs the de Gennes factor, $D G=(g-1)^{2} J(J+1)$, in Fig. 5 . This shows that the FM transition due to the itinerant $3 d$-electrons of the $\mathrm{Cr}$ ions, as well as the localized $4 f$-moments of the rare-earth ions scale with the de Gennes factor. In addition, both transition temperatures converge at the ferrimagnetic transition found for $\mathrm{GdCrSb}_{3}$. The result provides further evidence of the behavior of the ferrimagnetic phase in which the Gd and Cr moments strongly interact, and become anti-aligned at the same temperature. The inset to Fig. 5 shows the paramagnetic Curie temperature, averaged over the three principle axis, for each member in the series. Although a general downward trend is observed, it 
is not clear if there is a strong correlation between the de Gennes factor and the paramagnetic Curie temperature, and a doping study on the rare-earth site is underway in order to clarify this relationship. However, the general trend shows that both the rare-earth magnetic ordering temperature and the paramagnetic Curie temperature scale with the de Gennes factor.

\section{Conclusions}

While above $T_{C}=86 \mathrm{~K}, \mathrm{GdCrSb}_{3}$ has very similar magnetic and electrical resistivity properties to those of the light rare-earth chromium antimonides, at lower temperatures very different behavior is found. Both the magnetic susceptibility vs temperature and the magnetization vs field indicate that the system enters a ferrimagnetic phase below $86 \mathrm{~K}$. It is within this ferrimagnetic phase that the anisotropy of the electrical resistivity is clearly evident with insulating behavior found along the stacking axis (the $a$-axis), and metallic behavior found within the $b$ - $c$ plane. In addition, the de Gennes scaling of itinerant FM and localized rare-earth ordering transition temperatures for $R \mathrm{CrSb}_{3}$ converge to the $86 \mathrm{~K}$ transition of $\mathrm{GdCrSb}_{3}$. These are all indications of a ferrimagnetic phase in which the itinerant $3 d$ and localized $4 f$ moments are heavily interacting, and anti-aligned.

\section{Acknowledgements}

Scott McCall has been very helpful during many discerning discussions. The authors would like to thank Mike Torelli and Donavan Hall for their generous help. We would also like to thank Julia Loewecke for her insightful conversa- 
tions. This work was funded by the In House Research Program at the National High Magnetic Field Laboratory, which was funded by the NSF, Cooperative Agreement No. DMR-9527035.

This work was performed under the auspices of the U.S. Department of Energy by the University of California, Lawrence Livermore National Laboratory under Contract W-7405-Eng-48.

\section{References}

[1] M. Brylak, W. Jeitschko, Zeitschrift fuer Naturforschung B (Chem. Sci.) B50 (6) (1995) 899-904.

[2] M. Ferguson, R. Hushagen, A. Mar, J. Alloys. Comp. 249 (1) (1997) 191-8.

[3] N. P. Raju, J. E. Greedan, M. J. Ferguson, A. Mar, Chem. of Mater. 10 (1998) 3630.

[4] D. D. Jackson, M. Torelli, Z. Fisk, Phys. Rev. B 65 (2002) 014421.

[5] E. Granado, H. Martinho, M. S. Sercheli, P. G. Pagliuso, D. D. Jackson, M. Torelli, J. Lynn, C. Rettori, Z. Fisk, S. B. Oseroff, Phys. Rev. Lett. 89 (2002) 107204.

[6] K. Hartjes, W. Jeitschko, M. Brylak, J. of Magn. Magn. Mater. 173 (1-2) (1997) $109-16$.

[7] M. Leonard, S. Saha, N. Ali, J. Appl. Phys. 85 (8) (1999) 4759-61.

[8] M. Leonard, I. Dubenko, N. Ali, J. Alloys. Comp. 303-304 (2000) 265-9.

[9] L. Deakin, M. J. Ferguson, A. Mar, Chem. Mater. 13 (2001) 1407.

[10] D. D. Jackson, Z. Fisk, J. of Magn. Magn. Mater. 256 (2003) 106.

[11] I. S. Dubenko, P. Hill, N. Ali, J. Appl. Phys. 89 (11) (2001) 7326-7328.

[12] L. Deakin, A. Mar, Chem. Mater. 15 (2003) 3343. 
[13] E. Konig, Magnetic Properties of Coordination and Organo-metallic Transition Metal Compounds, Springer Verlag, Berlin, 1966.

[14] N. River, A. Mensah, Physica B 91 (1977) 85.

[15] K. Taylor, M. Darby, Physics of Rare Earth Solids, Chapman and Hall Ltd., 11 New Fetter Lane, London, 1972.

[16] D. D. Jackson, M. E. Torelli, Z. Fisk, single crystals of $\mathrm{NdCrSb}_{3}$ and $\mathrm{PrCrSb}_{3}$ have also been investigated. 
Fig. 1. Field cooled magnetization for the three principle axis of $\mathrm{GdCrSb}_{3}$. The insets show (a) the $R T \mathrm{Sb}_{3}$ crystal structure, and (b) the high temperature fit to a Curie-Weiss law.

Fig. 2. Magnetization as a function of field for $\mathrm{GdCrSb}_{3}$ at $5 \mathrm{~K}$. The inset shows the magnetization along the $a$-axis at $10 \mathrm{~K}$ up to $31 \mathrm{~T}$.

Fig. 3. Electrical resistivity of $\mathrm{GdCrSb}_{3}$ normalized to room temperature. The insets show (a) $d \rho_{b} / d T$ with a broad peak at the ferrimagnetic Curie temperature, and (b) the $T^{3 / 2}$ behavior of the resistivity below $86 \mathrm{~K}$.

Fig. 4. a) Inverse magnetic susceptibility along the $c$-axis for GdVSb 3 with an applied field of $10 \mathrm{kG}$, with the low temperature susceptibility displayed in the inset showing the AFM transition at $5 \mathrm{~K}$. b) The in-plane electrical resistivity of $\mathrm{GdVSb}_{3}$ normalized to room temperature.

Fig. 5. The de Gennes scaling for $R \mathrm{CrSb}_{3}$ which show both the Curie temperature due to the $\mathrm{Cr}$ ions, and the low temperature ordering due to the rare-earth ions. Both transition temperatures converge at the $86 \mathrm{~K}$ transition for $R=\mathrm{Gd}$. Inset shows the paramagnetic Curie temperature, averaged over the three principle axis, which also scales with the de Gennes factor (data from $[4,10,16]$ ). 
Table 1

Hight temperature Curie-Weiss parameters for $\mathrm{GdTSb}_{3}(T=\mathrm{Cr}, \mathrm{V})$. The error in $\chi_{0}$ is $10 \%$. Sample dimensions of $\mathrm{GdVSb}_{3}$ precluded the measurement along the $a$-axis.

$\mathrm{GdCrSb}_{3}$

\begin{tabular}{|c|c|c|c|}
\hline axis & $\chi_{0}\left(10^{-3} \mathrm{emu} / \mathrm{mol}\right)$ & $\mu_{e f f}\left(\mu_{B} /\right.$ f.u. $)$ & $\theta(\mathrm{K})$ \\
\hline$a$ & -1.27 & $7.89 \pm 0.13$ & $-90 \pm 12$ \\
\hline$b$ & -4.60 & $10.3 \pm 0.3$ & $-88 \pm 11$ \\
\hline$c$ & -2.45 & $9.45 \pm 0.25$ & $-86 \pm 13$ \\
\hline \multicolumn{4}{|c|}{$\mathrm{GdVSb}_{3}$} \\
\hline$b$ & -0.57 & $8.25 \pm .01$ & $21.5 \pm 0.1$ \\
\hline$c$ & -.085 & $8.33 \pm .01$ & $-22.9 \pm 0.1$ \\
\hline
\end{tabular}




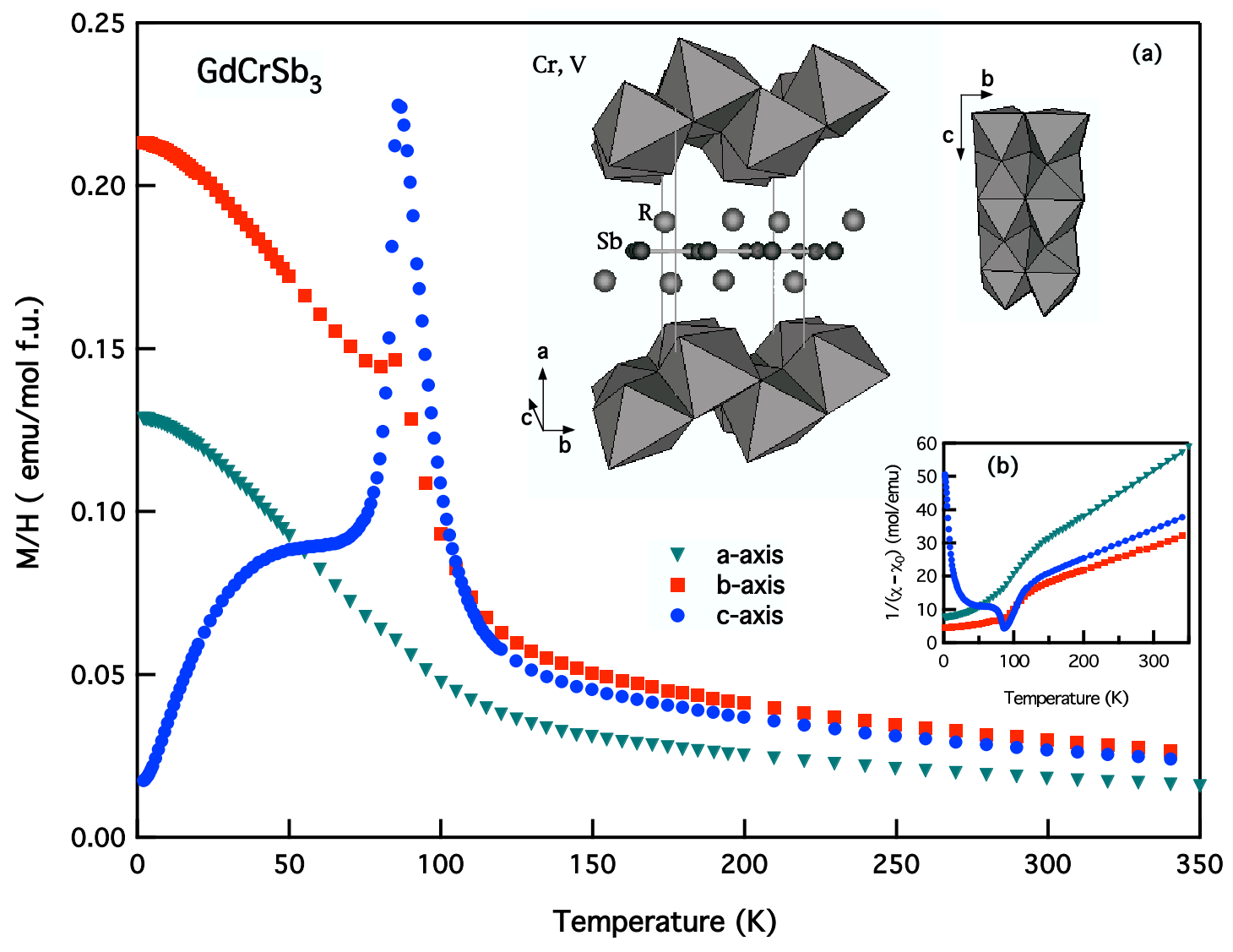




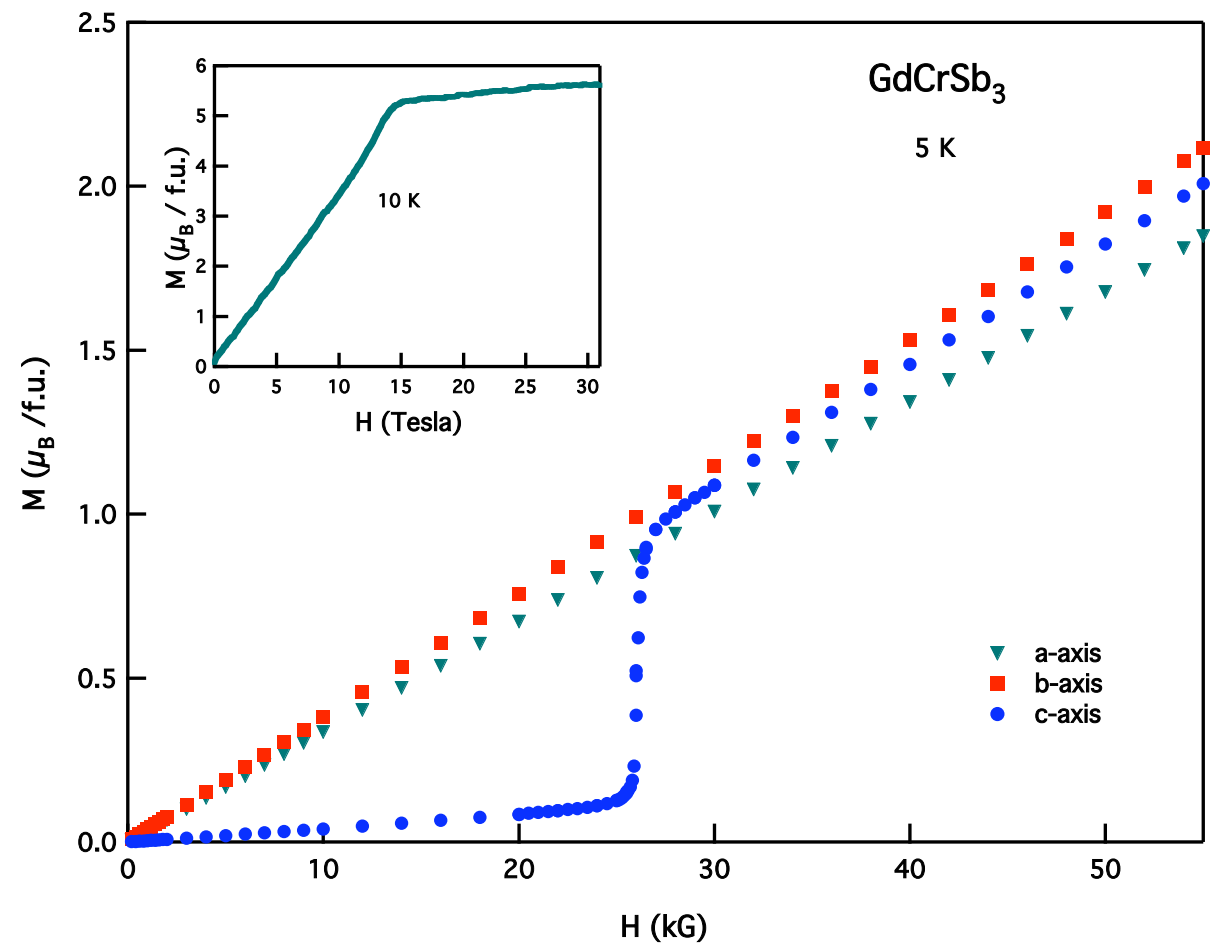




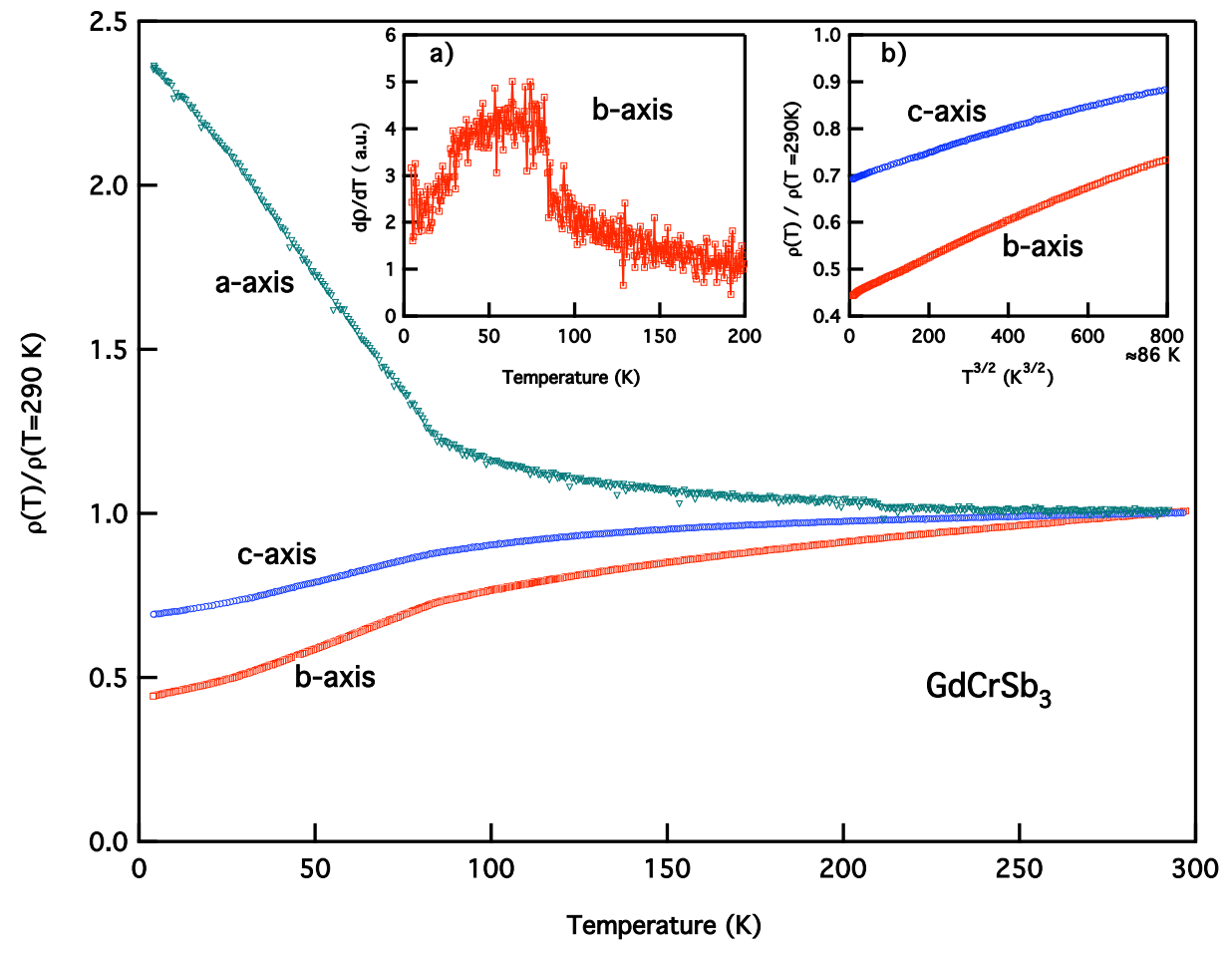




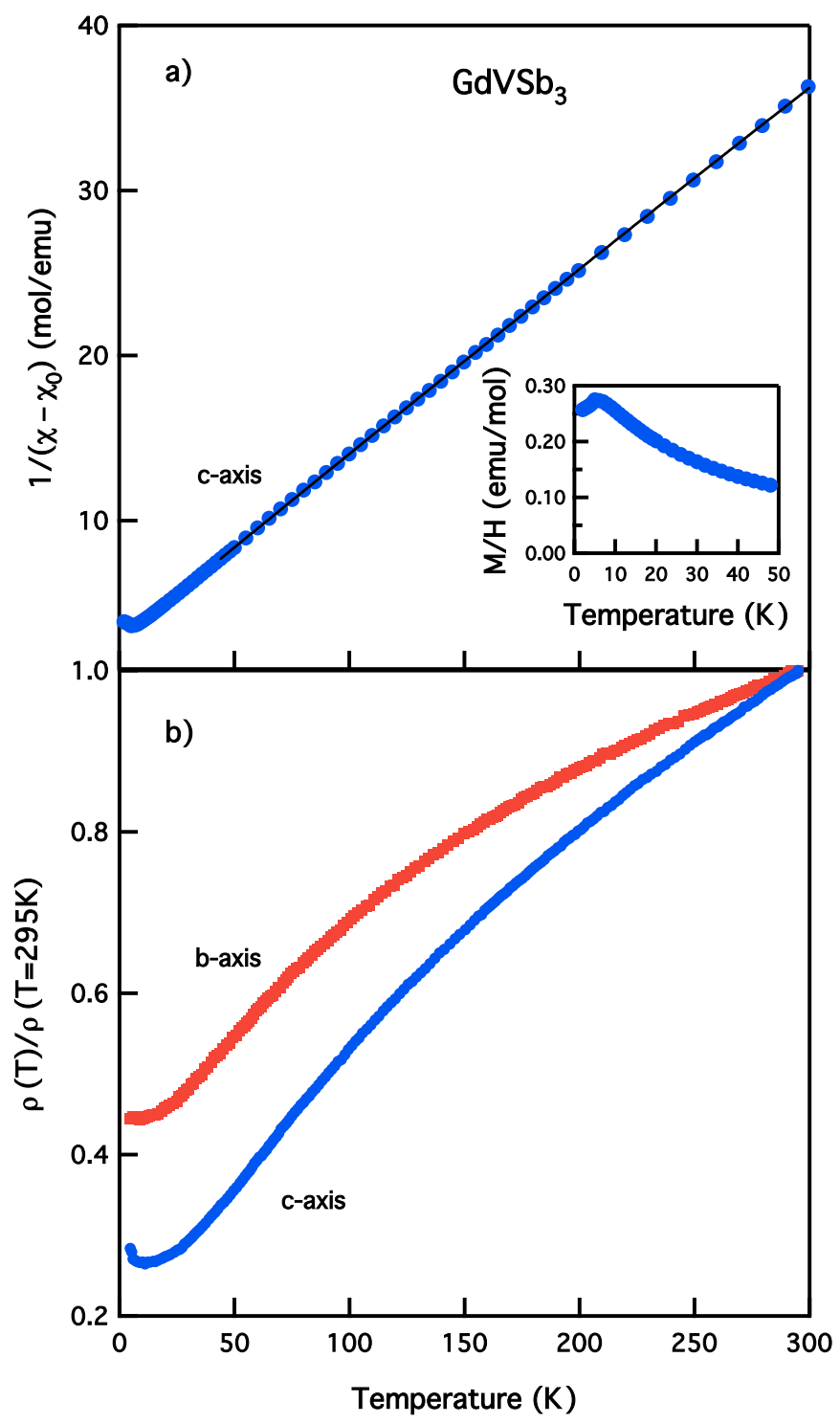




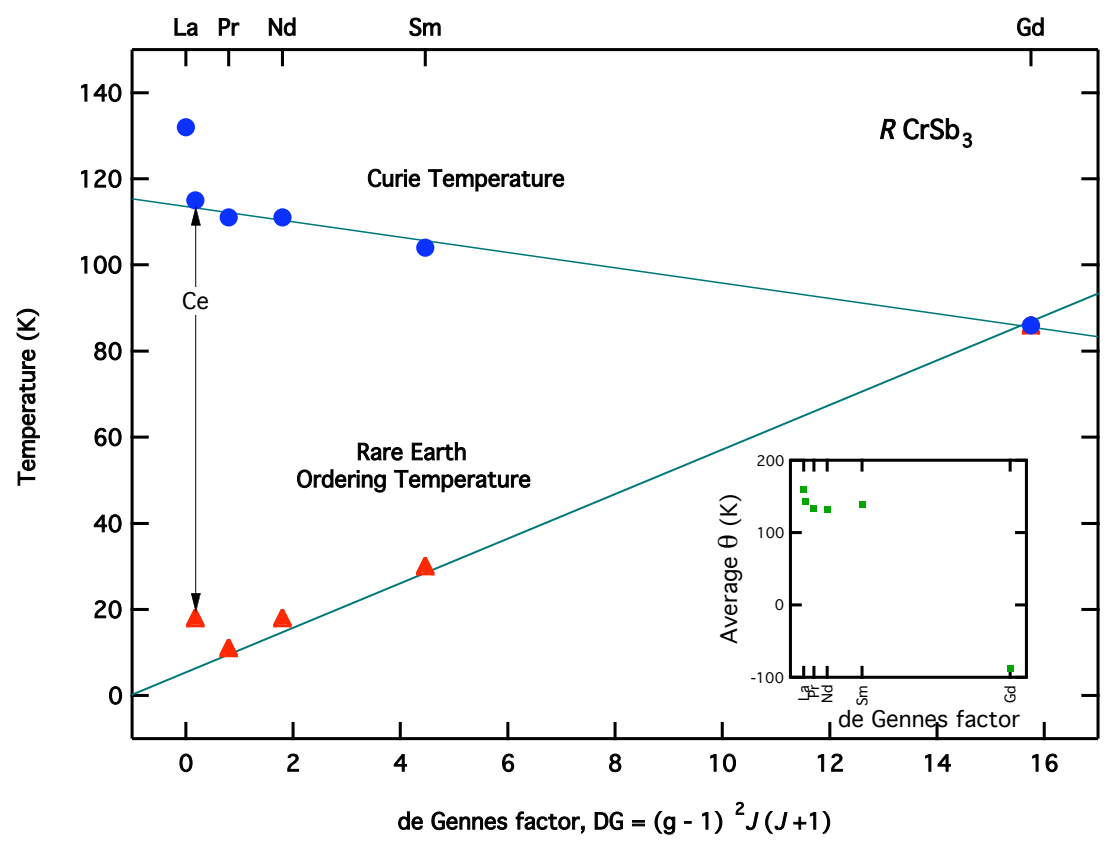

